\title{
Comparison of Maximal Lingual Pressure Generation During Isometric Gross and Fine Sensorimotor Tasks in Healthy Adults
}

\author{
Nicole Rogus-Pulia, PhD, ${ }^{a, b}$ Kim Churness, MS, ${ }^{a, b}$ Jacqueline Hind, MS, ${ }^{a, b}$ \\ Ronald Gangnon, PhD, ${ }^{c}$ Kelsey Banaszynski, MS, ${ }^{a, d}$ JoAnne Robbins, PhD $^{a, b}$
}

From the ${ }^{a}$ William S. Middleton Memorial Veterans Hospital, Geriatric Research Education and Clinical Center, Madison, WI; ${ }^{b}$ Division of Gastroenterology and Hepatology, Department of Medicine, University of Wisconsin School of Medicine and Public Health, Madison, WI; and Departments of ${ }^{C}$ Biostatistics and Medical Informatics and Population Health Sciences, and ${ }^{d}$ Nutrition, University of Wisconsin, Madison, WI.

\begin{abstract}
Objectives: To (1) compare 2 distinct isometric lingual press tasks, fine sensorimotor versus gross sensorimotor, at multiple sensor locations in relation to age and sex; and (2) provide a normative data set using a lingual-strengthening device.

Design: Cohort study.

Setting: University.

Participants: Healthy men and women $(\mathrm{N}=71$; age range, $21-82 \mathrm{y})$ recruited from the community.

Interventions: Participants were stratified by age and sex and divided into 3 age groups. Participants completed, in random order, 2 isometric tasks: (1) fine sensorimotor: tongue press maximally and discreetly against each of 5 sensors; and (2) gross sensorimotor: tongue press maximally against all 5 sensors simultaneously.

Main Outcome Measures: Primary outcome was maximum isometric pressure in hectopascals (hPa). Secondary outcomes were time to reach peak pressure (s) and pressure gradient $(\mathrm{hPa} / \mathrm{s})$.

Results: Maximum pressures were significantly lower in those of older age for both fine and gross sensorimotor lingual tasks $(P<.01)$, with the front and back sensors showing the greatest decline (35\% and 45\%, respectively). Pressure differences between tasks $(P=.0012)$ resulted in the fine sensorimotor task generating higher pressures at the front sensor for all age groups. However, the gross sensorimotor task generated faster maximum pressures at all sensor locations for all age groups. For both sensorimotor tasks, subjects of older age as a whole generated less steep pressure gradients $(P<.001)$.

Conclusions: Age-related decline in tongue strength is greater at the anterior and posterior tongue. Results indicate a simpler gross sensorimotor task may be more beneficial for targeting timing as a biomechanical parameter during therapy, and the fine sensorimotor task may be more beneficial for targeting strength.
\end{abstract}

Archives of Physical Medicine and Rehabilitation 2015; $\mathbf{0} \mathbf{\square}-\mathbf{\square} \mathbf{\square}$

(c) 2015 by the American Congress of Rehabilitation Medicine

Presented in part to the Dysphagia Research Society, March 6, 2014, Nashville, TN; and the American Speech-Language-Hearing Association, November 15, 2013, Chicago, IL.

Supported by the United States Department of Agriculture (grant no. NRI2007-2234). The views and content expressed in this article are solely the responsibility of the authors and do not necessarily reflect the position, policy, or official views of the Department of Veteran Affairs or the U.S. Government.

This manuscript was prepared at the William S. Middleton Memorial Veteran Affairs Hospital, Madison, WI; Geriatric Research, Education and Clinical Center manuscript \#2014-016.

Disclosures: Robbins has received patent licenses or has patent licenses pending from Bracco Diagnostics, Inc, and Swallow Solutions, LLC. Hind reports financial support from Swallow Solutions, LLC, outside the submitted work. The other authors have nothing to disclose.
Dysphagia affects as many as 15 million Americans, ${ }^{1}$ with the prevalence increasing with advancing age. Dysphagia affects $22 \%$ of those older than 50 years, and up to $55 \%$ of adults in aged-care settings. $^{2-4}$ Consequences of oropharyngeal dysphagia may include aspiration pneumonia, malnutrition, dehydration, weight loss, and reduced quality of life. Understanding the biomechanics and kinetics that contribute to dysphagia will allow for improved management and prevention of its negative influence on overall health. 
The tongue is the major propulsive driving force in healthy oropharyngeal swallowing. Age-related changes in tongue sensorimotor function decrease bolus propulsion in individuals older than 60 years, which prolongs oral transit time and contributes to dysphagia in the elderly. ${ }^{5,6}$ Changes in oral-motor function are due at least in part to sarcopenia. ${ }^{7}$ Relative to the oropharyngeal musculature, this diminished tongue muscle mass may lead to reduced tongue strength.

Tongue strength correlates with oral and pharyngeal transit times, as well as the percentage of oral residue, ${ }^{8}$ and is a predictor of oral phase swallowing impairment. ${ }^{9}$ Following tonguestrengthening protocols, maximum isometric lingual pressures increased in relatively young and older adults. ${ }^{10-15}$ Carryover to swallowing function has been observed through increased peak swallowing pressures, ${ }^{10,12}$ reduced pharyngeal residue, ${ }^{12,15}$ faster oral transit times, ${ }^{12}$ decreased penetration/aspiration scale scores ${ }^{16}$ (less airway invasion), ${ }^{12,15}$ and improved swallow-specific quality of life. ${ }^{12,13,15,17}$ While initial findings support the use of musclestrengthening programs, optimal approaches for building tongue strength remain to be elucidated. Various regions of tongue function have been analyzed relative to pressure generation ${ }^{6,10,12,18-21}$; however, patterns of tongue pressure generation at multiple sensor locations comparing gross and fine sensorimotor lingual isometric tasks have not been examined. Complex biomechanics and kinetics of the tongue are critical for optimizing lingual strength (for intrinsic and extrinsic muscles) and require further study regarding the most effective ways to engage different tongue regions for isometric lingual-strengthening protocols. It remains unknown how sensorimotor task instructions, which may influence cognitive understanding, lingual neuromotor control, and sensorimotor feedback, may affect timing, pressure building, and maximum isometric pressures generated at varying tongue regions.

The purpose of this study was to compare 2 isometric lingual tasks (gross vs fine) in relation to age and sex. This study also sought to provide normative data across the healthy adult life span. Five specific hypotheses were tested: (1) The maximum isometric pressure will be lower in those of older age during both sensorimotor tasks. (2) The time to reach maximum isometric pressure will be lower in those of older age for both sensorimotor tasks. (3) A higher maximum isometric lingual pressure will be generated during the fine sensorimotor task. ${ }^{22,23}$ Since the greatest range of forces may be produced by recruiting more motor units, ${ }^{23}$ isolated pressure production and motor units at 1 region of the tongue are likely to generate more strength than when activating and diffusing muscle fibers across the tongue (eg, whole tongue press). (4) A faster time to reach maximum isometric pressure occurs during the gross sensorimotor tasks. (5) The pressure gradient becomes less steep in those of older age for both sensorimotor tasks. ${ }^{18,24-26}$ Decreased innervation of muscle fibers in human skeletal muscle, particularly type II fast twitch fibers, ${ }^{26}$ along with slowed movements ${ }^{24}$ and slower swallowing ${ }^{25}$ in older adults, suggests that pressure building may be slower.

\section{Methods}

\section{Participants}

Seventy-one healthy men and women were recruited into 3 sexmatched age groups a priori: group 1 (youngest), group 2 (middle), and group 3 (oldest) (table 1). These groups were sex matched in that the same number of men and women was recruited into each group.
Table 1 Subject demographics

\begin{tabular}{llllll}
\hline & \multicolumn{2}{c}{ Age $(y)$} & & \multicolumn{2}{c}{ Sex } \\
\cline { 2 - 3 } \cline { 5 - 6 } Group No. & Range & Mean & & Men & Women \\
\hline 1 & $21-40$ & 25 & & 12 & 11 \\
2 & $41-60$ & 50 & & 12 & 12 \\
3 & $61-82$ & 68 & & 12 & 12 \\
\hline
\end{tabular}

Subjects were recruited through community flyers. Inclusion criteria were (1) age $\geq 21$ years; (2) self-reported normal swallowing; (3) consuming a general diet; and (4) able to provide informed consent. Exclusion criteria were (1) a history of neurologic insult/disease; (2) a history of swallowing problems; (3) the presence of food allergies; and (4) a history of or treatment for head/neck cancer.

\section{Pressure instrumentation and placement}

Fine and gross sensorimotor tasks were measured using the Madison Oral Strengthening Therapeutic device. ${ }^{\mathrm{a}}$ The instrument consists of a mouthpiece connected to a dedicated netbook computer for measuring lingual pressures in hectopascals (hPa). The mouthpiece has 5 air-filled sensors for quantification of pressure individually (single sensor) or simultaneously (multiple sensors) (fig 1). The mouthpiece is 1 size and custom molded by the clinicians to the hard palate, with the anterior sensor at the alveolar ridge. Sensors are equal distance between one another. Given different palatal configurations, the remaining sensors, while still in the areas of left, right, and back of the palate, may be slightly different between subjects, but within subject relative relationships are maintained. Because the mouthpiece is custom molded, intrasubject sensor placement is consistent across all swallows. The sampling rate is $100 \mathrm{~Hz}$.

\section{Isometric lingual testing procedure}

Subjects completed 2 types of isometric lingual tasks: gross smotor and fine sensorimotor. The gross sensorimotor task was defined as pressing the whole tongue against all 5 sensors

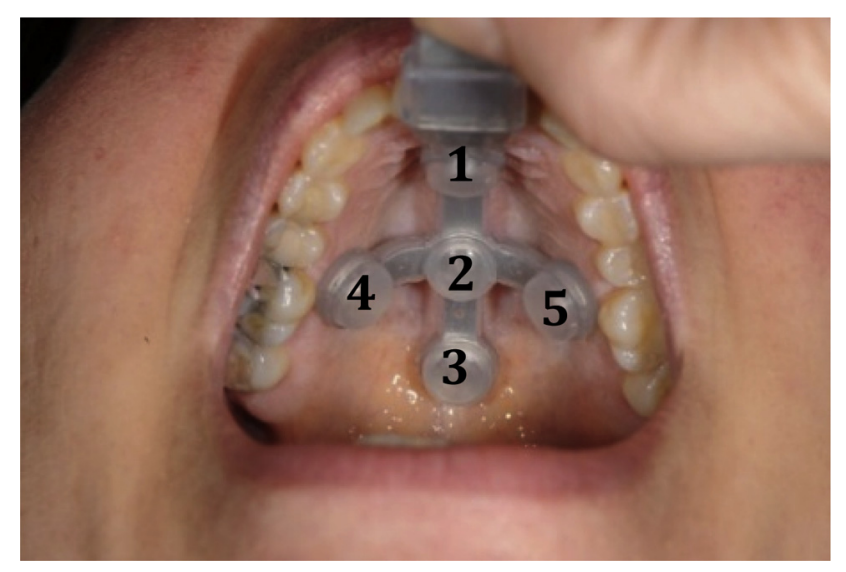

Fig 1 Photograph of Madison Oral Strengthening Therapeutic device custom-fit mouthpiece that consists of 5 pressure sensors: 1 , anterior; 2 , middle; 3 , posterior; 4, right; 5 , left. 
Time to Reach Peak

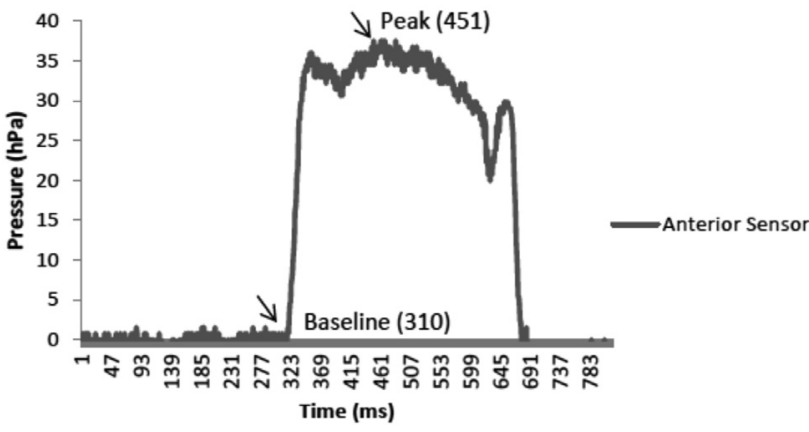

Fig 2 Example waveform of time to reach peak pressure in milliseconds. For each repetition, the first maximum peak pressure data point was subtracted by the first upstroke of pressure from baseline to determine the time to reach peak pressure (eg, Peak 451ms-Baseline $310 \mathrm{~ms}=141 \mathrm{~ms}$ ).

simultaneously with maximum effort. Subjects were instructed to, "Take your whole tongue and press it against the roof of your mouth." The fine sensorimotor task was defined as pressing discreetly with maximal effort on 1 individual sensor with the corresponding region of tongue. Subjects were instructed to, "Take the back of your tongue (front, etc) and press it against the back (front, etc) sensor." There were 3 sets of maximum presses for gross motor with 3 press repetitions within each set, followed by 3 sets of fine sensorimotor tasks with 3 press repetitions per set. One minute of rest was given between each of the 3 sets. Each maximum press repetition was held for 2 seconds, with 2- to 3second rests between repetitions. Fifteen-minute rest periods were provided between gross and fine motor tasks. Subjects received positive coaching to complete the repetitions. The order of sets (fine vs gross) was randomized/counterbalanced across all subjects. The sensor pressed during the fine sensorimotor task was randomized as well.

\section{Measurements}

Maximum isometric pressures $(\mathrm{hPa})$ were obtained by recording the 1 repetition maximums, defined as the load one can bear with maximal effort to complete a single repetition consistently. ${ }^{27}$ The time to reach peak pressure, known as the duration between onset of pressing and achievement of maximal pressure, was recorded in milliseconds. The measurement was calculated by subtracting the time the pressure reached maximum peak from the time of first pressure upstroke from baseline (fig 2). Gradient pressures were calculated by dividing maximum isometric pressures $(\mathrm{hPa})$ by the times to reach peak pressure (s) (fig 3).

\section{Data analysis}

Mixed-effects linear regression models were used to model maximum isometric pressure, (log-transformed) time to peak pressure and pressure gradient as a function of task (individual or whole tongue), sensor location (front, middle, back, right, left), age, and sex. Primary analyses used age as a continuous variable, while descriptive analyses used age as a categorical variable matching the recruitment strata. Dependence between measurements at different sensors on a single press, and dependence between measurements at 2 sensors on 2 presses in the same subject were modeled using

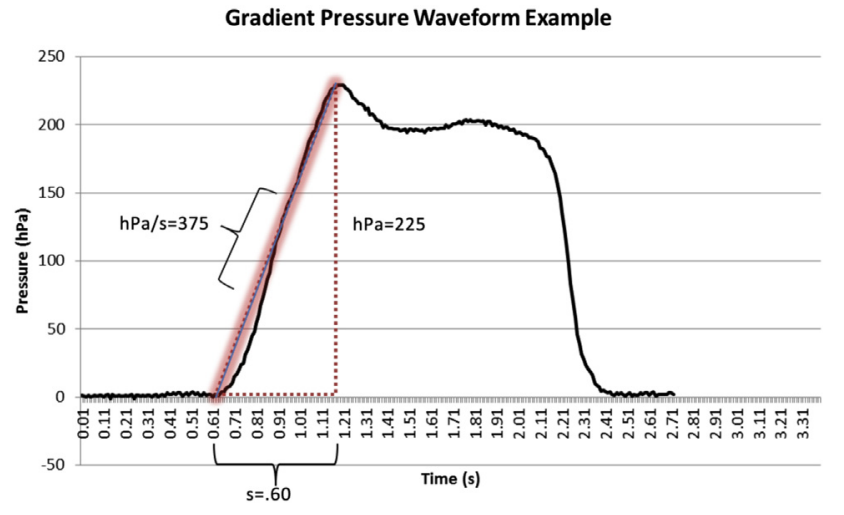

Fig 3 Pressure gradient was determined by dividing maximum isometric lingual pressure by time to reach peak pressure. Time to reach peak pressure was converted from milliseconds to seconds for this equation. This gradient pressure waveform is an example from a front sensor fine s-motor press (eg, $225 \mathrm{hPa} \div .60 \mathrm{~s}=375 \mathrm{hPa} / \mathrm{s})$.

unstructured variance-covariance matrices. Analyses were conducted using PROC MIXED in SAS version 9.3. ${ }^{\mathrm{b}}$

\section{Results}

\section{Task effects}

\section{Maximum pressure}

Comparison of 1 repetition maximums between the 2 sensorimotor lingual tasks revealed significantly higher pressures at the front sensor during the fine sensorimotor task in all 3 age groups (youngest, $P<.0007$; middle, $P<.0001$; and oldest, $P<.0001$ ) and higher pressures at the middle sensor during the gross sensorimotor task in all 3 age groups (youngest, $P<.0001$; middle, $P<.0001$; and oldest, $P<.0001)$. No other consistent patterns were found at the left, right, and back sensors between lingual sensorimotor tasks across age groups (table 2).

\section{Time to reach peak pressure}

For all subjects, the gross sensorimotor task produced faster peak pressures compared with fine sensorimotor tasks (table 3).

\begin{tabular}{|c|c|c|c|c|c|c|}
\hline \multirow{2}{*}{$\frac{\text { Age }}{\text { Task }}$} & \multicolumn{2}{|c|}{$\begin{array}{c}\text { Group } 1 \\
\text { (Youngest } \\
\text { Group) }\end{array}$} & \multicolumn{2}{|c|}{$\begin{array}{c}\text { Group } 2 \\
\text { (Middle } \\
\text { Group) }\end{array}$} & \multicolumn{2}{|c|}{$\begin{array}{c}\text { Group } 3 \\
\text { (Oldest } \\
\text { Group) }\end{array}$} \\
\hline & Gs-m & Fs-m & Gs-m & Fs-m & Gs-m & Fs-m \\
\hline Front S & 65.2 & $111.3^{*}$ & 41.7 & $61.5^{*}$ & 26.6 & $33.9^{*}$ \\
\hline Middle S & $49.7^{*}$ & 40.4 & $33.5^{*}$ & 28.7 & 22.6 & 20.3 \\
\hline Back S & 54.8 & 54.1 & 31.7 & 33.3 & 18.3 & 20.5 \\
\hline Left $S$ & 43.2 & $48.8^{*}$ & 34.2 & 34.1 & $27.1^{*}$ & 23.9 \\
\hline Right S & 31.6 & 35.0 & 25.6 & 25.8 & 20.8 & 19.0 \\
\hline
\end{tabular}

NOTE. Age/task/sensor interaction, $P<.001$.

Abbreviations: Fs-m, fine sensorimotor; Gs-m, gross sensorimotor; S, sensor.

* Significantly increased maximum pressures generated between sensorimotor tasks. 
Table 3 Time to reach peak pressure (s)

\begin{tabular}{|c|c|c|c|c|c|c|}
\hline \multirow{2}{*}{$\frac{\text { Age }}{\text { Task }}$} & \multicolumn{2}{|c|}{$\begin{array}{c}\text { Group } 1 \\
\text { (Youngest } \\
\text { Group) }\end{array}$} & \multicolumn{2}{|c|}{$\begin{array}{l}\text { Group } 2 \\
\text { (Middle } \\
\text { Group) }\end{array}$} & \multicolumn{2}{|c|}{$\begin{array}{c}\text { Group } 3 \\
\text { (Oldest } \\
\text { Group) }\end{array}$} \\
\hline & Gs-m & Fs-m & Gs-m & Fs-m & Gs-m & Fs-m \\
\hline Front $S$ & $0.97^{*}$ & 1.36 & $1.02^{*}$ & 1.26 & 1.08 & 1.16 \\
\hline Middle S & $0.52^{*}$ & 0.64 & $0.57^{*}$ & 0.64 & 0.62 & 0.65 \\
\hline Back S & 0.51 & 0.53 & $0.52^{*}$ & 0.58 & $0.54^{*}$ & 0.63 \\
\hline Left $S$ & $0.82^{*}$ & 0.94 & $0.87^{*}$ & 0.97 & 0.92 & 1.01 \\
\hline Right S & 0.50 * & 0.60 & $0.53^{*}$ & 0.64 & $0.56^{*}$ & 0.69 \\
\hline
\end{tabular}

NOTE. Age/task/sensor interaction, $P<.04$.

Abbreviations: Fs-m, fine sensorimotor; Gs-m, gross sensorimotor; $S$, sensor.

* Significantly decreased time to reach peak pressure between sensorimotor tasks.

Although this trend was observed at all sensors for all age groups, not all time differences between tasks reached significance. Significant time differences occurred at all sensors except the back in the youngest group $(P<.04)$, at all sensors in the middle group $(P<.04)$, and at the back and right sensors in the oldest group $(P<.04)$.

\section{Pressure gradient}

For all age groups, pressure gradient comparisons between tasks revealed steeper pressure gradients during the fine sensorimotor task at the front sensor $(P<.04)$, and steeper pressure gradients during the gross sensorimotor task at the middle sensor $(P<.04)$. A different pattern occurred at the right and left sensors in the middle and oldest groups where pressure gradients were significantly steeper during the gross sensorimotor task compared with the fine sensorimotor task $(P=.04)$. No significant differences were observed between sensorimotor tasks at the posterior sensor for any age group (table 4).

\section{Age effects}

\section{Maximum pressure}

Maximum isometric pressures were significantly lower in the middle and oldest age groups at all sensors during both fine and gross sensorimotor tasks $(P<.01)$. Interestingly, pressures

Table 4 Pressure gradient $(\mathrm{hPa} / \mathrm{s})$

\begin{tabular}{|c|c|c|c|c|c|c|}
\hline \multirow{2}{*}{$\frac{\text { Age }}{\text { Task }}$} & \multicolumn{2}{|c|}{$\begin{array}{c}\text { Group } 1 \\
\text { (Youngest } \\
\text { Group) }\end{array}$} & \multicolumn{2}{|c|}{$\begin{array}{l}\text { Group } 2 \\
\text { (Middle } \\
\text { Group) }\end{array}$} & \multicolumn{2}{|c|}{$\begin{array}{c}\text { Group } 3 \\
\text { (Oldest } \\
\text { Group) }\end{array}$} \\
\hline & Gs-m & Fs-m & Gs-m & Fs-m & Gs-m & Fs-m \\
\hline Front $S$ & 67.03 & $82.09^{*}$ & 40.69 & $48.91^{*}$ & 24.69 & $29.14^{*}$ \\
\hline Middle S & $95.67^{*}$ & 63.59 & $59.06^{*}$ & 44.56 & $36.46^{*}$ & 31.22 \\
\hline Back S & 107.91 & 101.71 & 60.53 & 57.45 & 33.95 & 32.45 \\
\hline Left $S$ & 52.87 & 52.03 & $39.46^{*}$ & 35.06 & $29.45^{*}$ & 23.62 \\
\hline Right S & 63.28 & 58.70 & $48.63^{*}$ & 40.08 & $37.37^{*}$ & 27.37 \\
\hline
\end{tabular}

NOTE. Age/task/sensor interaction, $P<.001$.

Abbreviations: Fs-m, fine sensorimotor; Gs-m, gross sensorimotor; $S$, sensor.

* Significantly increased pressure gradient between sensorimotor tasks. decreased at similar rates between the 2 sensorimotor tasks in those of older age. For both sensorimotor tasks, the front and back sensors decreased the most $(\sim 35 \%-45 \%)$, followed by the middle $(\sim 30 \%)$ and left and right sensors $(\sim 20 \%-30 \%)$ (see table 2).

\section{Time to reach peak pressure}

The time to reach maximum pressures slowed in those of older age; however, it did not reach statistical significance $(P>.1)$ at any sensor $(<10 \%$ change every $20 \mathrm{y})$ (see table 3 ).

\section{Pressure gradient}

The pressure gradient became significantly less steep in those of older age at all sensors during both sensorimotor tasks $(P<.001)$. Of interest, pressure gradients decreased at similar rates between the fine and gross sensorimotor tasks with increasing age. The pressure gradient dropped most in older subjects at the back sensor ( $\sim 45 \%)$, followed by the front $(\sim 40 \%)$, middle $(\sim 30 \%-40 \%)$, and left and right $(20 \%-30 \%)$ sensors (see table 4$)$.

\section{Sex effects}

\section{Maximum pressure}

A significant sex-by-sensor interaction $(P=.001)$ was driven by men producing higher pressures at the front, right, left, and middle sensors; women produced higher pressures at the back sensor.

\section{Time to reach peak pressure}

The time to reach peak pressure was not significantly affected by $\operatorname{sex}(P=.55)$.

\section{Pressure gradient}

A significant sex-by-sensor interaction $(P<.002)$ was driven by men producing steeper pressure gradients at the front, middle, left, and right sensors, and women producing steeper pressure gradients at the back sensor.

\section{Discussion}

\section{Maximum pressures and age}

This study contributes to an understanding of tongue function during isometric tasks over the normal life span. The first major finding was that isometric lingual pressures decreased in those of older age for both fine and gross sensorimotor tasks. This replicates previous studies ${ }^{18,25,28-30}$ that found lingual strength to be lower in older adults, and likely reflects the age-related loss (sarcopenia) of skeletal muscle mass, strength, and function. ${ }^{31}$ Although multifactorial mechanisms driving sarcopenia are not clearly understood, increased physical activity has been shown to reverse or modify development of this condition, ${ }^{32}$ leading to the development of lingual-strengthening programs ${ }^{10-12,14}$ designed to improve swallowing function. ${ }^{10,12,33,34}$

Anterior to posterior progressive activation of lingual musculature produces the propulsion necessary for bolus transport during oropharyngeal swallowing. ${ }^{35-37}$ Pressure generation decreased most in those of older age at the anterior and posterior tongue regions compared with the other tongue regions analyzed (middle, left, right). Although muscles of the tongue and their histopathologic changes in the aging process are not clearly understood, our 

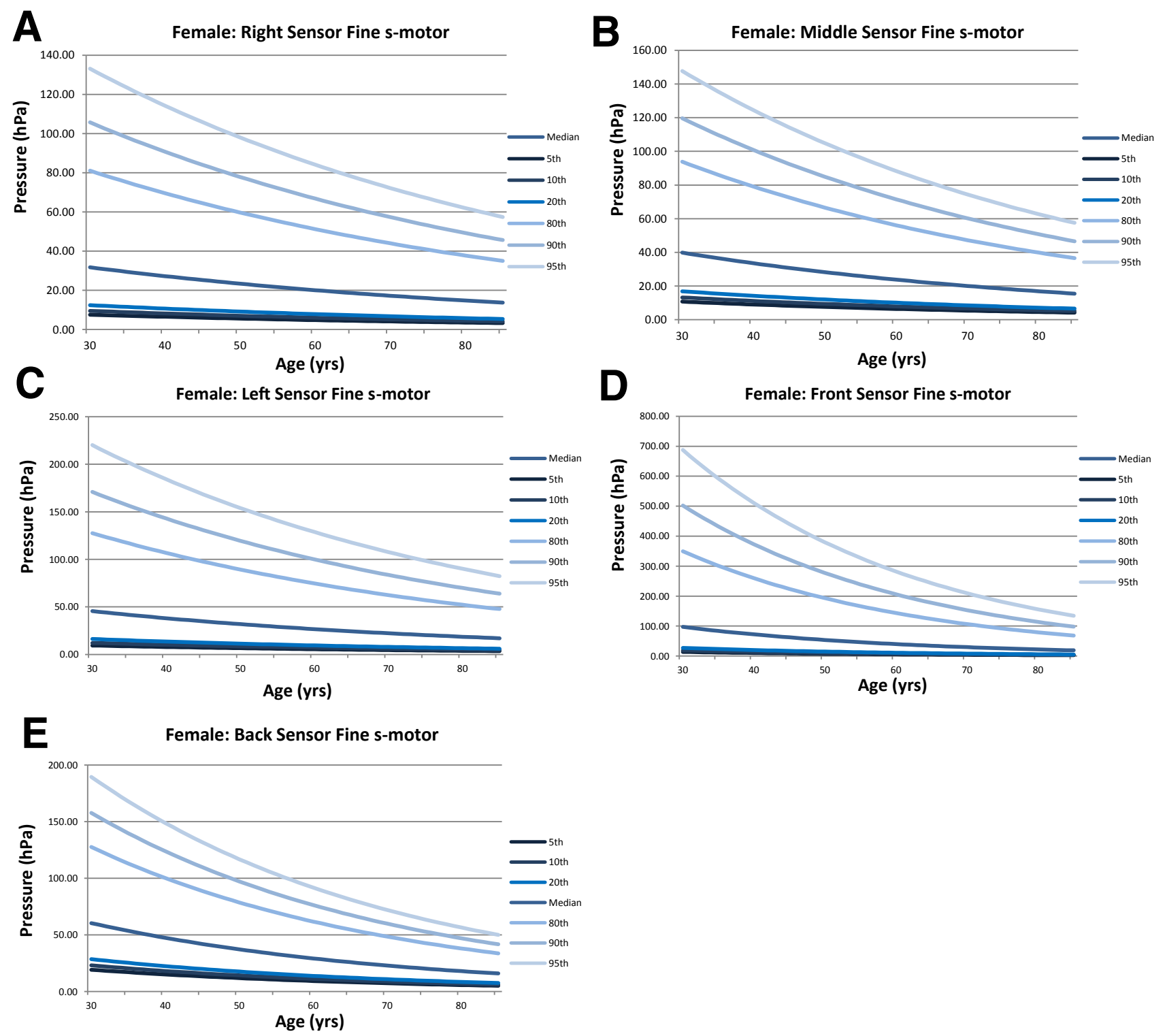

Fig 4 Means and percentile ranks of maximum isometric lingual pressures generated at 5 sensor locations by 35 healthy women during the fine sensorimotor (s-motor) press. Women: (A) Right sensor. (B) Middle sensor. (C) Left sensor. (D) Front sensor. (E) Back sensor.

findings support the idea that tongue-to-palate pressures at those regions may be more predisposed to the effects of sarcopenia influencing motor and sensory aspects.

\section{Maximum pressures: anterior sensor}

Analyzing pressure produced at specific sensors between smotor tasks resulted in a consistent trend. Subjects in all age groups produced significantly higher pressures at the front sensor when performing the fine sensorimotor task. This is not surprising because the anterior tongue is made up of more collagen and elastic connective tissue fiber ${ }^{38}$ than the posterior tongue and contains $71 \%$ type II (fast twitch) muscle fibers, ${ }^{22}$ allowing for flexibility, range of motion, and finely graded movements that are more voluntarily controlled. Volitional control of the anterior tongue may permit channeling of more power at the front sensor during fine sensorimotor tasks rather than diffusing pressure across all tongue regions such as during gross sensorimotor tasks. Using fine sensorimotor tasks for generating pressure in therapy may better isolate and strengthen the anterior tongue muscles necessary for anchoring and driving adequate and efficient bolus propulsion. ${ }^{28}$

\section{Maximum pressures: posterior sensor}

In contrast to the anterior sensor, pressures generated at the posterior sensor were similar between sensorimotor tasks in all age groups. The posterior tongue, consisting of up to $66 \%$ type I (slow twitch) muscle fibers, ${ }^{22}$ is mainly used in larger, slower movements such as positioning and forming tongue shape. It contributes to more neurally hardwired pharyngeal events, such as the brainstem-mediated pharyngeal response. ${ }^{39}$ Therefore, the posterior tongue functions in a "more automated" fashion, making volitional control of precise fine sensorimotor movements less distinct from gross sensorimotor movements during pressure generation. Individuals who have sensory deficits or cognitive 

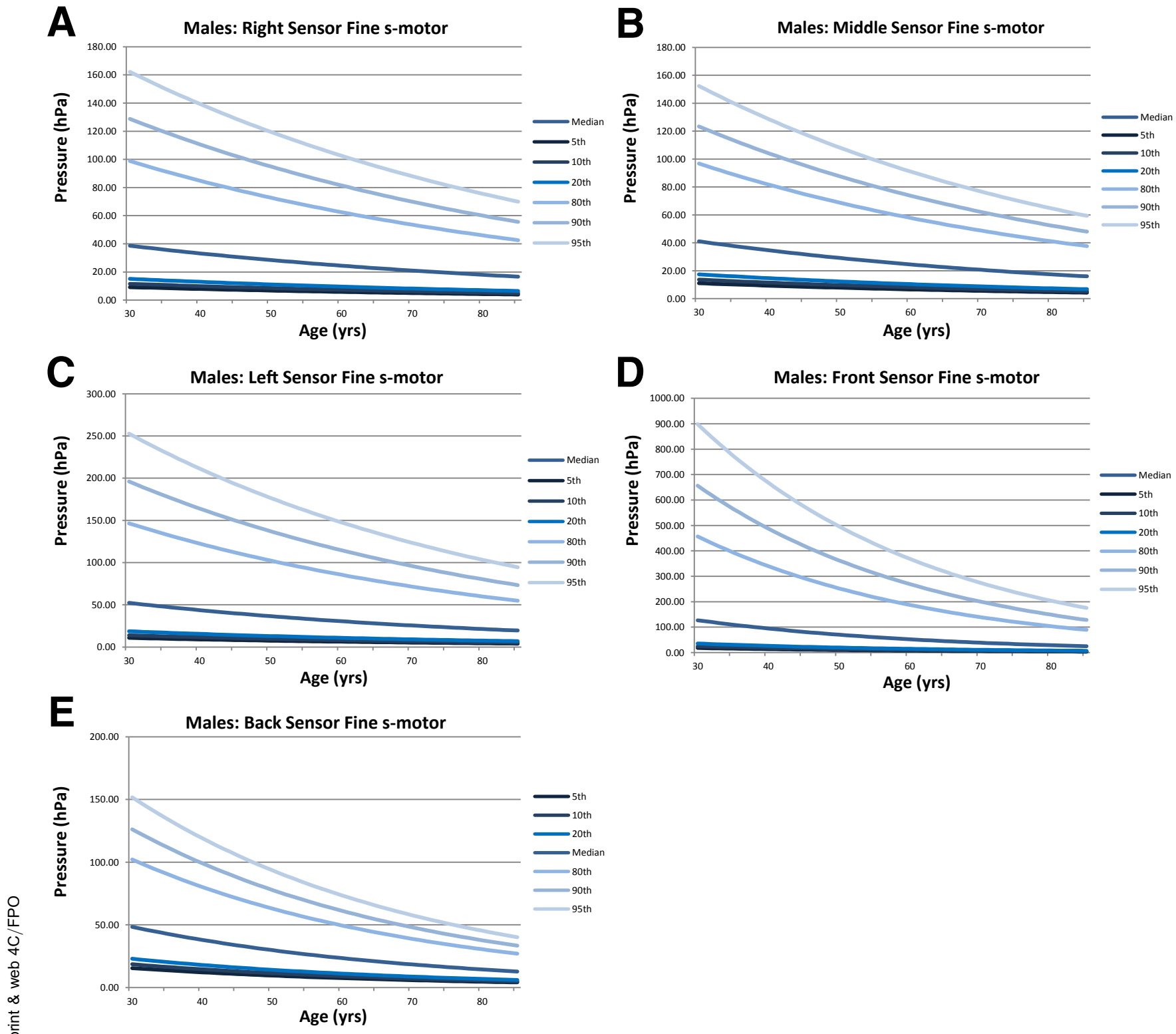

Fig 5 Means and percentile ranks of maximum isometric lingual pressures generated at 5 sensor locations by 36 healthy men during the fine sensorimotor (s-motor) press. (A) Right sensor. (B) Middle sensor. (C) Left sensor. (D) Front sensor. (E) Back sensor.

limitations may benefit from use of a less coordinated whole tongue (gross sensorimotor) task. It also is possible that subjects may learn to have more precise control of pressure on the posterior sensor with practice. Since measures of posterior tongue strength are similar between sensorimotor tasks, clinicians have more flexibility in choosing which sensorimotor task to use in therapy.

\section{Time to peak pressures and sensorimotor task differences}

Gross sensorimotor tasks produced faster times to reach peak pressures than fine sensorimotor tasks at all sensor locations in all age groups. When producing a new sensorimotor skill, individuals determine and refine somatosensory goals to establish the best motor commands to achieve their goal. ${ }^{40}$ Utilizing a gross sensorimotor task for producing faster maximum presses correlates with the need for less information processing by using a larger target size for pressure generation that is less complex to perform. Future research should address differences in the use of fine versus gross sensorimotor tasks in populations with oropharyngeal sensorimotor deficits to further understand these effects.

\section{Pressure gradient and age}

Along with a reduction in pressure generation found in persons of older age, the slope of pressure build (pressure gradient) was also found to become less steep in those of older age at all sensors for both sensorimotor tasks, with the largest reduction at the posterior $(45 \%)$ and anterior sensors $(40 \%)$. Hori et $\mathrm{al}^{35(\mathrm{p} 13)}$ suggested that the "slope of tongue pressure may have a relationship with the transport of bolus." A more gradual pressure gradient at the posterior tongue region with healthy aging may allow healthy individuals time to coordinate airway closure with slower bolus movement through the hypopharynx. ${ }^{25}$ However, for patients who 

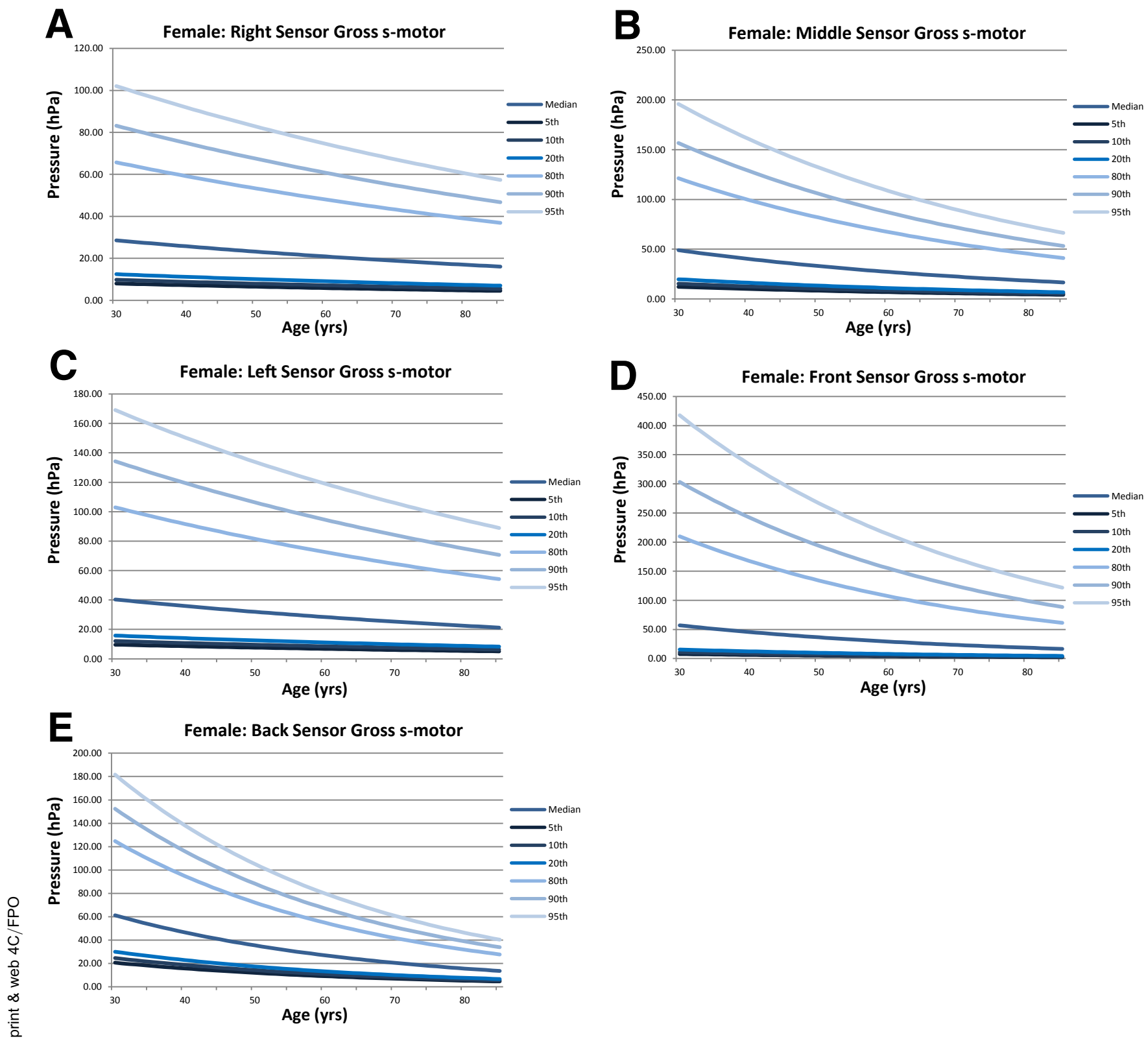

Fig 6 Means and percentile ranks of maximum isometric lingual pressures generated at 5 sensor locations by 35 healthy women during the gross sensorimotor (s-motor) press. Women: (A) Right sensor. (B) Middle sensor. (C) Left sensor. (D) Front sensor. (E) Back sensor.

have difficulty with strength and coordination of elevating the posterior tongue to the hard palate for adequate bolus hold and propulsion, focusing on pressure gradient may have potential clinical benefits, although more research is needed to assess clinical applicability.

The gross sensorimotor task generated steeper pressure gradients at the right and left sensors, and both tasks generated similar pressure gradients at the posterior sensor. Lateral borders and the posterior region of the tongue seal the bolus against the hard palate before initiation of the pharyngeal swallow. A gross sensorimotor task may result in better lingual motor control for bolus containment and warrants further research.

\section{Pressure generation and sex}

Men produced higher maximum isometric pressures at the anterior, middle, left, and right sensors, which is consistent with previous studies. $^{20,21,30,41}$ This has been attributed to greater muscle mass in men. ${ }^{28}$ We found that men have steeper pressure gradients at the front, middle, left, and right sensors. Although men reached higher maximum isometric pressures, they still maintained a faster rate of pressure build. This indicates that larger muscle mass may play a role in pressure building. It is unclear why women produced higher pressures and steeper pressure gradients at the posterior sensor. As hypothesized by Ney et al, ${ }^{42}$ perhaps more voluntary events become "uncoupled" from the "neurally hardwired" brainstem pharyngeal response. If true, then the glossopharyngeal nerve and its sensory inclusion in the more automated swallow response may change how motor movements of the posterior tongue are controlled compared with movements of the anterior and middle tongue involved in more volitional acts and innervated predominately by the trigeminal and facial nerves. Further research focused on morphological tongue differences is needed. 

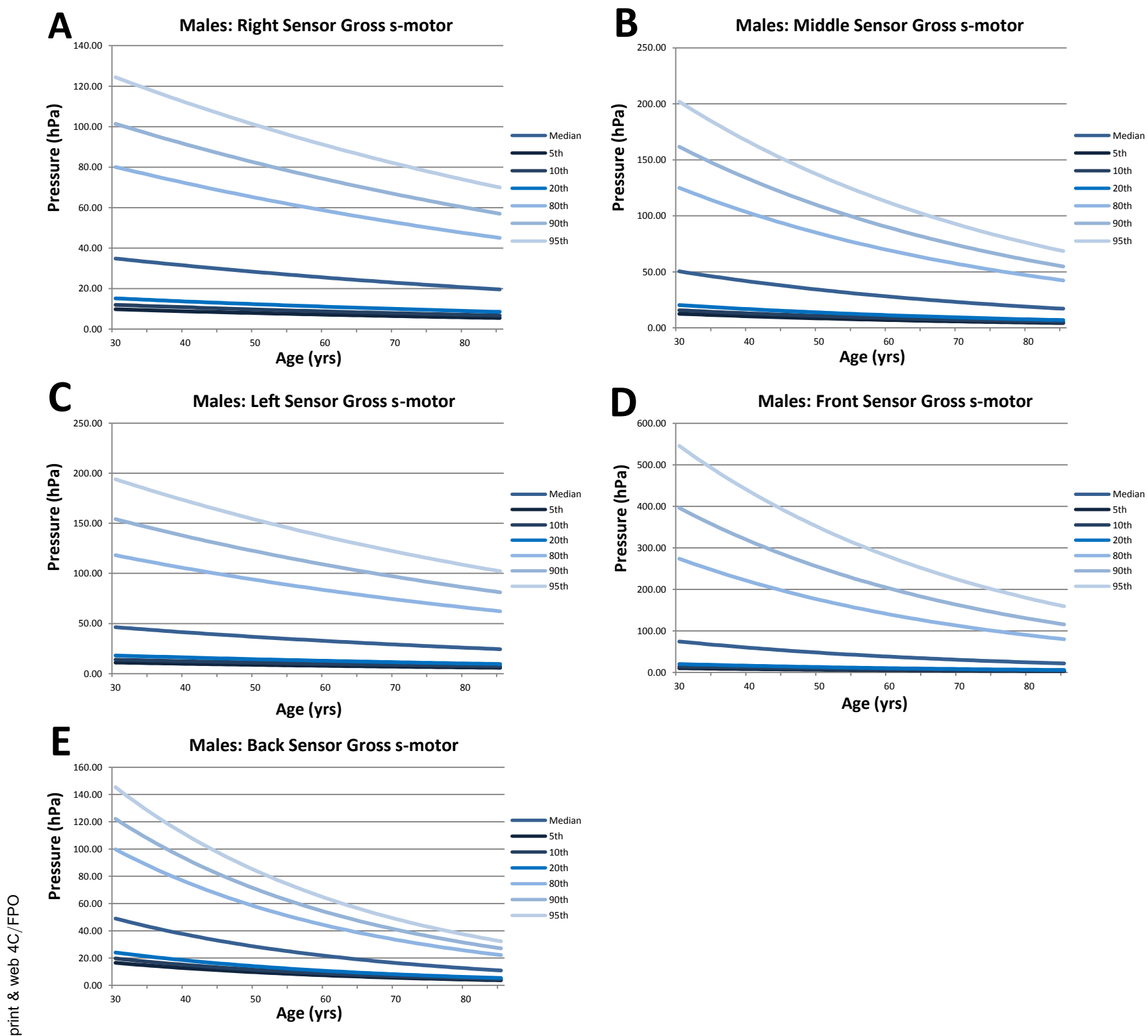

Fig 7 Means and percentile ranks of maximum isometric lingual pressures generated at 5 sensor locations by 36 healthy men during the gross sensorimotor (s-motor) press. (A) Right sensor. (B) Middle sensor. (C) Left sensor. (D) Front sensor. (E) Back sensor.

\section{Study limitations}

Validation that subjects isolated targeted regions of their tongue against 1 specific sensor during the fine sensorimotor task was unavailable because lingual movements were not visualized instrumentally. This study also was unable to adjust for the mouthpiece length of the Madison Oral Strengthening Therapeutic device in relation to the subjects' palate size. Although the anterior sensor was placed at the alveolar ridge in all subjects, the mouthpiece's single-size structure places the posterior sensor at arbitrary locations relative to palate and tongue length. Therefore, objective measurements gathered at the posterior sensor, in comparison to the anterior sensor, may need to be interpreted with caution. Intersubject differences in motor strategies for generating pressure against tongue sensors may also have occurred. This may explain some findings of the study, such as sex-based pressure-by-sensor differences, which cannot be fully explained by the current data. While time to peak pressure is likely an effortbased, response-time task, subjects were not provided with instructions to complete the task quickly. This may have contributed to the nonsignificant findings in this study.

\section{Conclusions}

Isometric lingual strength and pressure gradients decreased in those of older age for both gross and fine sensorimotor lingual tasks in this cohort of healthy adults. While trends were observed in both sensorimotor tasks, there were significant differences in pressure gradient, lingual strength, and time to reach peak pressure between tasks that may support 1 task as more beneficial than another depending on which biomechanical parameters are 
targeted in therapy (ie, strength vs timing). Additional work to address use of a gross versus a fine sensorimotor lingual task in specific dysphagia patient groups is necessary for development of the most beneficial strengthening programs.

Normative data for fine (figs 4 and 5) gross sensorimotor (figs 6 and 7) pressures at various tongue regions serve as a foundation for understanding maximum isometric lingual pressures based on age and sex. Assessing pressure is 1 way of understanding the biomechanics of lingual functioning that may lead to susceptibility for oropharyngeal dysphagia. It is yet to be determined whether rehabilitative and preventive measures using fine or gross sensorimotor tasks may facilitate prevention of the negative sequelae of aging effects, dysphagia, or both.

\section{Suppliers}

a. Madison Oral Strengthening Therapeutic device; Swallow Solutions, LLC.

b. PROC MIXED in SAS version 9.3; SAS Institute Inc.

\section{Keywords}

Deglutition disorders; Pressure; Rehabilitation; Tongue

\section{Corresponding author}

Nicole Rogus-Pulia, PhD, 2500 Overlook Terrace, Madison GRECC (11G), Madison, WI 53705. E-mail address: npulia@ wisc.edu.

\section{Acknowledgments}

The authors would like to thank Jenny Christensen, BA, for her assistance with manuscript editing, and Naomi Humpal, BS, for her assistance with data analyses and interpretation.

\section{References}

1. Simmons K. Dysphagia management means diagnosis, exercise, reeducation. JAMA 1986;255:3209-10. 3212.

2. Howden CW. Management of acid-related disorders in patients with dysphagia. Am J Med 2004;117(Suppl 5A):44S-8S.

3. Holland G, Jayasekeran V, Pendleton N, Horan M, Jones M, Hamdy S. Prevalence and symptom profiling of oropharyngeal dysphagia in a community dwelling of an elderly population: a self-reporting questionnaire survey. Dis Esophagus 2011;24:476-80.

4. Cichero JA, Altman KW. Definition, prevalence and burden of oropharyngeal dysphagia: a serious problem among older adults worldwide and the impact on prognosis and hospital resources. Nestle Nutr Inst Workshop Ser 2012;72:1-11.

5. Niederman MS, Fein AM. Pneumonia in the elderly. Clin Geriatr Med 1986;2:241-68.

6. Sonies BC, Parent LJ, Morrish K, Baum BJ. Durational aspects of the oralpharyngeal phase of swallow in normal adults. Dysphagia 1988;3:1-10.

7. Evans WJ. What is sarcopenia? J Gerontol A Biol Sci Med Sci 1995; 50(Spec No):5-8.

8. Lazarus C, Logemann J, Pauloski B, et al. Swallowing and tongue function following treatment for oral and oropharyngeal cancer. J Speech Hear Res 2000:1011-23.
9. Clark HM, Henson P, Barber W, Stierwalt J, Sherrill M. Relationships among subjective and objective measures of tongue strength and oral phase swallowing impairments. Am J Speech Lang Pathol 2003;12: 40-50.

10. Robbins J, Gangnon RE, Theis SM, Kays SA, Hewitt AL, Hind JA. The effects of lingual exercise on swallowing in older adults. J Am Geriatr Soc 2005;53:1483-9.

11. Lazarus C, Logemann JA, Huang CF, Rademaker AW. Effects of two types of tongue strengthening exercises in young normals. Folia Phoniatr Logop 2003;55:199-205.

12. Robbins J, Kays SA, Gangnon RE, et al. The effects of lingual exercise in stroke patients with dysphagia. Arch Phys Med Rehabil 2007; $88: 150-8$.

13. Carroll WR, Locher JL, Canon CL, Bohannon IA, McColloch NL, Magnuson JS. Pretreatment swallowing exercises improve swallow function after chemoradiation. Laryngoscope 2008;118:39-43.

14. Clark HM, O'Brien K, Calleja A, Corrie SN. Effects of directional exercise on lingual strength. J Speech Lang Hear Res 2009;52: 1034-47.

15. Juan J, Hind J, Jones C, McCulloch T, Gangnon R, Robbins J. Case study: application of isometric progressive resistance oropharyngeal therapy using the Madison Oral Strengthening Therapeutic device. Top Stroke Rehabil 2013;20:450-70.

16. Rosenbek JC, Robbins JA, Roecker EB, Coyle JL, Wood JL. A penetration-aspiration scale. Dysphagia 1996;11:93-8.

17. McHorney C, Robbins J, Lomax K, et al. The SWAL-QOL and SWAL-CARE outcomes tool for oropharyngeal dysphagia in adults: III. Documentation of reliability and validity. Dysphagia 2002;17: 97-114.

18. Robbins J, Levine R, Wood J, Roecker EB, Luschei E. Age effects on lingual pressure generation as a risk factor for dysphagia. J Gerontol A Biol Sci Med Sci 1995;50:M257-62.

19. Crow HC, Ship JA. Tongue strength and endurance in different aged individuals. J Gerontol A Biol Sci Med Sci 1996;51:M247-50.

20. Trawitzki LV, Borges CG, Giglio LD, Silva JB. Tongue strength of healthy young adults. J Oral Rehabil 2011;38:482-6.

21. Youmans SR, Stierwalt JA. Measures of tongue function related to normal swallowing. Dysphagia 2006;21:102-11.

22. Stål P, Marklund S, Thornell LE, De Paul R, Eriksson PO. Fibre composition of human intrinsic tongue muscles. Cells Tissues Organs 2003;173:147-61.

23. Clamann P. Motor unit recruitment and the gradation of muscle force. Phys Ther 1993;73:830-43.

24. Gonzalez-Feire M, de Cabo R, Studenski S, Ferrucci L. The neuromuscular junction: aging at the crossroad between nerves and muscle. Front Aging Neurosci 2014;6:208.

25. Nicosia MA, Hind JA, Roecker EB, et al. Age effects on the temporal evolution of isometric and swallowing pressure. J Gerontol A Biol Sci Med Sci 2000;55:M634-40.

26. Lexell J. Human aging, muscle mass and fiber type composition. J Gerontol A Biol Sci Med Sci 1995;50(Spec No):11-6.

27. Fiatarone MA, Marks EC, Ryan ND, Meredith CN, Lipsitz LA, Evans WJ. High-intensity strength training in nonagenarians. Effects on skeletal muscle. JAMA 1990;263:3029-34.

28. Todd JT, Lintzenich CR, Butler SG. Isometric and swallowing tongue strength in healthy adults. Laryngoscope 2013;123:2469-73.

29. Vanderwegen J, Guns C, Van Nuffelen G, Elen R, De Bodt M. The influence of age, sex, bulb position, visual feedback, and the order of testing on maximum anterior and posterior tongue strength and endurance in healthy Belgian adults. Dysphagia 2013; 28:159-66.

30. Stierwalt JA, Youmans SR. Tongue measures in individuals with normal and impaired swallowing. Am J Speech Lang Pathol 2007;16: $148-56$.

31. Rosenberg IH. Sarcopenia: origins and clinical relevance. J Nutr 1997; 127(5 Suppl):990S-1S.

32. Montero-Fernández N, Serra-Rexach JA. Role of exercise on sarcopenia in the elderly. Eur J Phys Rehabil Med 2013;49:131-43. 
33. Rogus-Pulia N, Rusche N, Zielinski J, Hind J, Safdar N, Robbins JA. Effects of isometric progressive resistance oropharyngeal (I-PRO) therapy on dysphagia. In: Proceedings of the Dysphagia Research Society 22nd Annual Meeting; March 6-8, 2014; Nashville, TN.

34. Yeates EM, Molfenter SM, Steele CM. Improvements in tongue strength and pressure-generation precision following a tonguepressure training protocol in older individuals with dysphagia: three case reports. Clin Interv Aging 2008;3:735-47.

35. Hori K, Taniguchi $\mathrm{H}$, Hayashi $\mathrm{H}$, et al. Role of tongue pressure production in oropharyngeal swallow biomechanics. Physiol Rep 2013;1:e00167.

36. Stone M, Shawker TH. An ultrasound examination of tongue movement during swallowing. Dysphagia 1986;1:78-83.

37. Hamlet SL, Stone M, Shawker TH. Posterior tongue grooving in deglutition and speech: preliminary observations. Dysphagia 1988;3:65-8.
38. Miller JL, Watkin KL, Chen MF. Muscle, adipose, and connective tissue variations in intrinsic musculature of the adult human tongue. $\mathrm{J}$ Speech Lang Hear Res 2002;45:51-65.

39. Robbins J. Upper aerodigestive tract neurofunctional mechanisms: lifelong evolution and exercise. Head Neck 2011;33(Suppl 1): S30-6.

40. Vahdat S, Darainy M, Ostry DJ. Structure of plasticity in human sensory and motor networks due to perceptual learning. J Neurosci 2014;34:2451-63.

41. Youmans SR, Youmans GL, Stierwalt JA. Differences in tongue strength across age and gender: is there a diminished strength reserve? Dysphagia 2009;24:57-65.

42. Ney D, Weiss J, Kind A, Robbins J. Senescent swallowing: impact, strategies and interventions. Nutr Clin Pract 2009;24: 395-413. 